Received 8 May; accepted 10 July 1978

1. Hughes, G. M. \& Wiersma, C. A. G. J. exp. Biol. 37, 657-670 (1960)

2. Ikeda, K. \& Wiersma, C. A. G. Comp. Biochem. Physiol 12, 107-115 (1964).

3. Wiersma, C. A. G. \& Ikeda, K. Comp. Biochem. Physiol. 12, 509-525 (1964).

4. Stein, P. S. G. J. Neurophysiol, 34, 310-318 (1971)

5. Davis, W. J. J. Neurophysiol. 34, 274-288 (1971).

6. Burrows, M. \& Siegler, M. V.S. Nature 262, 222-224 (1976).

\section{Evidence for the functional involvement of carotenoids in the photoperiodic reaction of spider mites}

PHOTOPERIODISM in insects and mites depends on the presence of light-absorbing pigments, which are probably located in the central nervous system; photoperiodic responses are mediated by the direct action of light on these nervous elements ${ }^{1}$. Until now, pigments involved in photoperiodic processes such as diapause and polymorphism have not been identified. Different groups of pigments have been named by various authors as possible candidates for this function. A biologically active role based mainly on action spectra studies and the absorption characteristics of isolated pigments has been suggested for pterins ${ }^{2}$, carotenoids ${ }^{3}$, a combination of carotenoproteins, flavoproteins and haemoproteins ${ }^{4}$ and bile pigments $^{5}$. Carotenoids, pterins and flavins have also been proposed as possible photoreceptor pigments in studies on the spectral sensitivity of circadian rhythms ${ }^{6}$. According to Lees ${ }^{1}$, however, the usefulness of action spectra in identifying the light acceptor is limited, as spectra for photoperiodic action cannot at present be matched against absorption curves. A different approach was used by Zimmerman and Goldsmith ${ }^{7}$, who reared Drosophila on a carotenoid-free diet and showed that in one generation the sensitivity of the visual receptors was lowered by $3 \mathrm{log}$ units because of lack of rhodopsin. At the same time the photosensitivity of the rhythm of pupal eclosion in the cultures was not affected, from which it was concluded that the pigment involved in mediating light effects on the circadian rhythm in Drosophila is not a carotenoid derivative. We demonstrate here that carotenoids are functionally involved in the photoperiodic induction of diapause in the spider mite Tetranychus urticae using a method involving pigment mutants.

In $T$. urticae, only adult females may undergo a facultative diapause, induced by short-day photoperiods experienced during the larval and nymphal stages. With the test regime used $\left(10 \mathrm{~L}: 14 \mathrm{D}, 18^{\circ} \mathrm{C}\right)$ the different wild-type strains showed a $100 \%$ diapause $^{8}$. From a wild-type strain we have isolated seven different pigment mutants, all due to single recessive mutations which interfere with the uptake and oxidative metabolism of $\beta$-carotene in these mites 9 . It seemed that only the albino mutant showed a lowered diapause response, all other pigment mutants exhibiting $100 \%$ diapause in the above test regime. Four albino mutants, which arose spontaneously in different wild-type strains, all responded with a lowered diapause, although all four originated from wild-type strains which give $100 \%$ diapause in the test conditions. Complete diapause has never been observed in any albino strain; although highly variable in expression (diapause responses range over $0-80 \%$ in different tests), the percentage diapause in albino strains is always markedly reduced in comparison with wild type. Backcrossing of three of the albino mutants to wild type for 10 generations did not restore the capability to diapause. As crossing-over is thought to occur very frequently in these mites ${ }^{10}$, these findings are a strong indication for a functional involvement of the albino locus in the photoperiodic reaction of T. urticae.

Even stronger evidence for a functional connection between the presence of carotenoid pigments and diapause was obtained in other crossing experiments between wild type and albino. For these crosses use was made of the male haploidy (arrhenotoky) in $T$. urticae. The out-cross of the albino mutant to wild type gives hybrid daughters which are phenotypically wild type and diapause normally. Crossing these hybrid females to albino males produces a mixed female offspring, consisting of wild type and albino in a 1:1 ratio. Surprisingly, both these categories show a $100 \%$ diapause when tested in the photoperiodic regime mentioned above. Albino females of this offspring, after random mating with their brothers, which also consisted of a $1: 1$ ratio of wild type and albino, again gave rise to a mixed offspring of wild-type (hybrid) and albino females. From these, the wild-type females all diapaused on short-day treatment, whereas the albino daughters showed the same lowered diapause response as described for the different albino strains. From these crosses it seems that $100 \%$ diapause in albino is realised only when the albino daughters come from hybrid, phenotypically wild-type mothers. Apparently a maternal effect is responsible for the complete induction of diapause in albino females.

The lowered diapause response encountered in all albino strains and the fact that the capability to diapause could not be restored by a series of back-crosses to wild type, suggest that the albino locus exerts a pleiotropic effect, influencing both pigmentation and diapause induction. The discovery of the maternal effect in the crossing experiments described above confirms this supposition and leads us to conclude that carotenoids are functionally involved in diapause induction in $T$. urticae. With respect to pigmentation, a maternal effect, consisting of small red eyespots in albino males produced by wild-type hybrid females, has been demonstrated previously in these mites ${ }^{11}$. This showed that the egg of spider mites transmits a certain amount of carotenoids, which may be used for the formation of eyespots. From the experiments described above it could be concluded that these maternally derived carotenoids may also be utilised by the embryo in the formation of a photopigment concerned in the induction of diapause. Very small amounts of pigment would probably suffice for this function. A more elaborate account of these and other results will be published elsewhere.

\section{A. VEERMAN}

W. HELLE

Laboratory of Experimental Entomology,

University of Amsterdam,

Kruislaan 302,

1098 SM Amsterdam, The Netherlands

Received 15 June; accepted 28 July 1978

1. Lees, A. D. in Biochronometry (ed. Menaker, M.) 372-380 (National Academy Sciences Press, Washington, 1971).

2. L'Hélias, C. Bull. biol. Fr. Belg. 96, 187-198 (1962)

3. Lees, A. D. Nature 210, $986-989$ (1966).

4. Hayes, D. K. in Biochronometry (ed. Menaker, M.) 392-402 (National Academy Sciences Press, Washington, 1971).

Vuillaume, M. Seugé, J. \& Bergerard, J. Int. J Chronobiol 2, 181-188 (1974).

6. Frank, K. D. \& Zimmerman, W. F. Science 163, 688-689 (1969).

7. Zimmerman, W. F. \& Goldsmith, T. H. Science 171, 1167-1169 (1971)

8. Veerman, A. J. Insect Physiol. 23, 703-711 (1977).

9. Veerman, A. Comp. Biochem. Physiol. 47B, 101-116 (1974).

10. Zon, A. Q. van \& Helle, W. Entomologia exp. appl. 10, 69-74 (1967).

11. Ballantyne, G. H. Genetica 40, 289-323 (1969).

\section{Evidence for passive electrotonic interactions in red rods of toad retina}

SINCE Baylor, Fuortes and O'Bryan ${ }^{1}$ first demonstrated interactions between vertebrate photoreceptor cells, many types of inter-receptor influences have been described ${ }^{2-10}$. While some of these interactions are mediated by horizontal cells ${ }^{1,2,6}$, others apparently result from direct contact between the photoreceptors themselves ${ }^{1.3 .4 .7-10}$. Fain et al. ${ }^{10}$ reported that the red rods of the toad Bufo marinus were coupled by gap (electronic) junctions, and that single rods received detectable signals from other receptors over a retinal area of about $0.5 \mathrm{~mm}^{2}$. This 